\title{
Sperm parameters and DNA fragmentation of balanced chromosomal rearrangements carriers
}

\author{
Ewa Pastuszek ${ }^{1,2}$, Jolanta Kiewisz ${ }^{3}$, Patrycja M. Kulwikowska ${ }^{1,2}$, \\ Mariusz Lukaszuk ${ }^{1}$, Krzysztof Lukaszuk ${ }^{1,2}$
}

\author{
${ }^{1}$ INVICTA Fertility and Reproductive Center, Gdansk, Warsaw, Wroclaw, Poland \\ ${ }^{2}$ Department of Obstetrics and Gynecological Nursing, Faculty of Health Sciences, \\ Medical University of Gdansk, Poland \\ ${ }^{3}$ Department of Human Histology and Embryology, Faculty of Medical Sciences, \\ University of Warmia and Mazury, Olsztyn, Poland
}

\begin{abstract}
Introduction. Somatic chromosomal rearrangements that occur in infertile males are thought to be one of the major genetic factors influencing male infertility. The objective of this retrospective study was to evaluate sperm parameters in a group of patients with balanced translocations.

Material and methods. We analyzed semen of 84 balanced somatic translocation carriers [35 Robertsonian translocation (RT group) and 49 reciprocal translocation (RCT group)] and 57 men with normal karyotype (control group).

Semen samples were evaluated for sperm concentration, its motion parameters and vitality, round cell number (CASA) and DNA fragmentation index (TUNEL). Cytogenetic evaluation was also performed for each study participant.

Results. Sperm concentrations were lower when comparing the RT group to the control $(\mathrm{p}<0.001)$ and RCT groups $(\mathrm{p}<0.05)$. Occurrence of abnormal sperm concentration was more common among RT carriers $(74.3 \%)$ than in the other groups (42.9\% in RCT group and $28.1 \%$ in control group). The sperm progressive motility and vitality in RT carriers $(21.53 \%$ and $62.17 \%)$ were lower than in control group $(39.77 \%$ and $77.47 \%, \mathrm{p}<0.001$, respectively) and RCT carriers ( $31.47 \%$ and $76.17 \%, \mathrm{p}<0.001$, respectively). The RCT carriers and the control group did not differ in regard to sperm concentration, progressive sperm motility, motility grade D and sperm vitality. There were no significant differences in DNA fragmentation in carriers of both studied structural chromosomal rearrangements in comparison to subjects with normal karyotype.

Conclusions. RT carriers had significantly lower semen parameters in comparison to not only the subjects with normal karyotypes but also the RCT carriers. (Folia Histochemica et Cytobiologica 2015, Vol. 53, No. 4, 314-321)
\end{abstract}

Key words: Robertsonian translocation; reciprocal translocation; balanced chromosomal rearrangements; sperm parameters; DNA fragmentation

\section{Introduction}

It is estimated that approximately $15 \%$ of couples are infertile. Among the various causes of infertility, male factor is responsible for approximately $30-50 \%$

Correspondence address: P. Kulwikowska, M.Sc.

INVICTA Fertility and Reproductive Center

Rajska 10, 80-850 Gdansk

tel.: +48585858 800

e-mail: Patrycja.Kulwikowska@invicta.pl of cases. Nowadays, male infertility is considered as a multifactorial disease and identification of its etiology in an individual is critical for effective treatment.

Chromosomal rearrangements are thought to account for 2-8\% of male factor infertility [1]. Genetic changes influence fertility as they may affect a variety of processes including hormonal status, spermatogenesis, and sperm quality [2]. Genetic defects may cause the dysfunction of the hypothalamo-gonadal axis or affect development of male gonads and urogenital tract. Moreover, they may stop germ cell maturation 
or lead to the production of defective spermatozoa [3]. Effective chromosome segregation is essential for premeiotic divisions and meiosis, as sperm aneuploidy can arise at this stage due to segregation errors [4]. Understanding of male factor infertility requires a detailed knowledge of the biological and genetic factors needed for normal sperm development and function.

Chromosomal aberrations have been postulated to be one of the major genetic factors responsible for occurrences of male infertility [5, 6]. Somatic chromosomal rearrangements are found in infertile men approximately 10 times more often than in the general population [7]. The prevalence of balanced autosomal translocations in the population of infertile men has been reported to be in the range of 1.6-6.6\% [8-10]. Reciprocal translocations (RCT) are the replacement of genetic material between two or more chromosomes that does not result in the loss of genetic material [5]. A different kind of chromosomal rearrangement are Robertsonian translocations (RT) which involve the connection of two acrocentric chromosomes, in which two long (q) arms of each chromosome join to form a new chromosome and two short (p) arms are lost. This causes a condition of 45,XY but is considered balanced given that the lost short arms contain repetitive genetic material.

Balanced translocations (RCT and RT) are the most common chromosomal structural rearrangements identified in humans which do not usually cause phenotypic consequences, apart from possibility of infertility. Balanced translocation carriers may create unbalanced gametes leading to an increased risk of recurrent miscarriages or having children with congenital anomalies [11].

Sperm concentration is currently the most commonly examined parameter in relation to the prevalence of chromosomal rearrangements in infertile men. However, there have been no systematic studies of all sperm parameters including not only sperm concentration or motility but also vitality and DNA fragmentation in translocation carriers. Thus, the aim of this study has been to evaluate sperm parameters in a group of patients with balanced translocations (both $\mathrm{RCT}$ and RT) and compare them to the sperm parameters of unaffected donors with normal karyotypes.

\section{Material and methods}

Study population. The study protocol has been approved by the Institutional Review Board. All donors gave their informed consent for semen analysis and karyotyping.

This is a retrospective assessment of the data of patients treated between January 2009 and November 2014 at the
INVICTA Fertility Clinic (INVICTA, Gdansk, Poland). The study group included 84 men with balanced translocations (49 RCT and $35 \mathrm{RT}$ carriers). The mean age of participants was $34.65 \pm 4.72$ for RCT, $35.03 \pm 4.49$ for RT carriers, and $36.37 \pm 4.16$ years for control subjects ( $p>0.05)$. No statistically significant differences were found in terms of sexual abstinence. Round cell concentrations did not differ among the groups either.

Patients affected by other factors that could influence fertility (i.e. microdeletion of the AZF region, excessive alcohol intake, chronic or hallucinatory drug use, systemic disease, hereditary disorders) were excluded from the study.

Fifty seven men with normal karyotype and not meeting the exclusion criteria were randomly selected from among partners of women diagnosed with tubal factor, served as a control group. Since it is widely accepted [12] that $35 \%$ of the causes of infertility are female-related, $35 \%$ male-related, in $25 \%$ of the cases problem affects both partners, and in $5 \%$ of cases the cause remains unknown. We decided to use as the control group partners of women with a confirmed female factor, in order to get a population distribution similar to that of male factor infertility.

Semen analysis. Analysis of fresh semen samples were performed at the INVICTA Medical Laboratories in Gdansk, according to the guidelines of the World Health Organization [12]. Semen samples were collected by masturbation after 2-7 days of sexual abstinence. Samples were liquefied at room temperature for 60 minutes before analysis. The volume, $\mathrm{pH}$, color and viscosity were determined for each sample. The eosin test was performed to detect viable spermatozoa. Sperm counts and percentage motility were measured by computer assisted sperm analysis (CASA) system using the IVOS Hamilton Thorn Analyzer (Hamilton Thorne Inc., Beverly, Massachusetts, USA). At least one sperm analysis was recorded for all patients from all groups included in the study. In cases of patients with multiple analyses performed only data from the first sperm analysis were used.

Semen status was classified as follows: azoospermia (defined as the lack of sperm in the ejaculate) and severe oligozoospermia (sperm concentration $<5$ million $/ \mathrm{mL}$ ), oligozoospermia (sperm concentration 5-15 million/mL), normospermia (sperm concentration $>15 \mathrm{million} / \mathrm{mL}$ ), cryptozoospermia (spermatozoa absent from fresh preparations but observed in a centrifuged pellet). Sperm motility was classified as: A — rapid progressive motility; B — slow progressive motility; $\mathrm{C}$ - non progressive motility; $\mathrm{D}$ - immotility.

TUNEL assay. Due to the retrospective design of the study only one test was used for the evaluation of sperm DNA fragmentation. It was evaluated using a TUNEL assay kit (APO-DIRECT $^{\mathrm{TM}}$ Kit, BD Biosciences Pharmingen, San 
Diego, California, USA) in compliance with the manufacturer's instructions and specifications, with minor modifications. Briefly, spermatozoa were washed in phosphate-buffered saline (PBS) and placed onto the slide. Air-dried slides were fixed with $4 \%$ paraformaldehyde at room temperature. For permeabilization, the cells were incubated for $15 \mathrm{~min}$ at $4-8^{\circ} \mathrm{C}$ with $2 \%$ Triton X-100. Labeling, preceded by washing with Wash Buffer provided by the manufacturer, were performed by incubation for 1 hour in the dark at $37^{\circ} \mathrm{C}$ with labeling solutions [terminal deoxytransferase (TdT) enzyme, TdT reaction buffer, fluorescein isothiocynate-tagged deoxyuridine triphosphate nucleotides (FITC-dUTP), and distilled water]. After labeling, slides were rinsed in Rinsing Buffer and then counterstained with DAPI (4,6 diamidino-2-phenylindole). Each spermatozoon was considered to have either normal (blue nuclear fluorescence) or fragmented (partial or total green fluorescence) DNA. Sperm DNA fragmentation index was established as a final percentage of spermatozoa with fragmented DNA. The positive and negative control cells were provided in the APPDIRECT ${ }^{\mathrm{TM}}$ Kit (BD Biosciences Pharmingen, San Diego, California, USA). At least 300-400 cells in each sample were counted with the use of objective UPlanFl 60x NA 1.25 on Olympus BX62 epifluorescence microscope (Olympus, Tokyo, Japan). Each spermatozoon was photographed using CCD camera (Pixera Pro 600ES) and pictures were archived (Viewfinder ver. 3.0.1).

Cytogenetic evaluation. Cytogenetic examinations were performed on metaphases obtained from peripheral blood lymphocyte cultures using standard procedure [13]. Briefly, the $0.5 \mathrm{~mL}$ heparinized peripheral blood samples were cultured at $37^{\circ} \mathrm{C}$ for $72 \mathrm{~h}$ in RPMI-1640 Medium (Sigma-Aldrich, USA), supplemented with fetal bovine serum, (PAN-Biotech, Germany) and Lectins LF-7 (IBSS BIOMED SA, Poland). Culture was terminated $30 \mathrm{~min}$ after adding $10 \mu \mathrm{g} / \mathrm{mL}$ colchicines (Gibco, Life Technologies, Carlsbad, California, USA). Hypotension, fixation, trypsynization and Wright staining procedure were used to visualize G-banded chromosomes. Karyotypes were established at approximately $450-550$ bands resolution. At least 15 metaphases were analyzed for each patient. Images were captured using CytoVision software (ver. 3.9), and the karyotypes were designated according to International System for Chromosome Nomenclature (ISCN; 2013). Chromosome polymorphisms and variants or fragile sites were not considered in the description of abnormalities.

Statistical analysis. Statistical analyses were performed using GraphPad Prism (ver. 5.1, Graphpad Software Inc., San Diego, California, USA). Kruskal-Wallis test with post-hoc Dunn's Bonferroni test was used for comparisons among all analyzed groups and to test for significance. Additionally, the chi-square test was used to compare differences among the studied groups. Statistical significance was defined as $p<0.05$.

\section{Results}

Characteristics of patients, semen and sperm parameters of translocations carriers and the control group are shown in Table 1.

Results of lymphocyte karyotyping analysis among balanced translocation carriers are presented as Supplementary data. Examples of karyograms of the RCT and RT patients and DNA fragmentation of spermatozoa are presented in Figure 1.

No statistically significant differences were found between the study groups in terms of age, sexual abstinence, sperm volume, round cells concentration, sperm motility grade $\mathrm{C}$ and sperm DNA fragmentation index.

There were no significant differences in sperm concentration between the RCT carriers and the control group. However, diminished number of spermatozoa were observed in semen collected from RT carriers in comparison to the control group $(\mathrm{p}<0.001$; Figure 2A). Lower sperm concentrations were also observed for RT in comparison to RCT carriers $(\mathrm{p}<0.05$; Figure 2A).

In the course of our research, we found normal semen parameters in $25.7 \%$ of RT and $57.1 \%$ of RCT carriers (Figure 3). These rates were lower than in control subjects $(71.9 \%)$. Occurrences of abnormal sperm concentration were more common among carriers of a chromosomal rearrangement (42.9\% in RCT group and $74.3 \%$ in the RT group; Table 1) than among subjects with normal karyotype (28.1\%; Table 1). We found statistically significant differences in regard to occurrences of abnormal sperm concentration when comparing RT carriers to the control group $(\mathrm{p}<0.001)$ and to the RCT group ( $\mathrm{p}<0.05)$. Comparison of the RCT carriers group versus the control group yielded no significant difference.

Sperm vitality was reduced in samples from RT carriers in comparison to the control $(p<0.001)$ and RCT $(\mathrm{p}<0.001)$ carriers groups (Figure $2 \mathrm{~B})$. There were no statistical differences between RCT and control group.

Progressive sperm motility (grade A + B) was significantly lower $(\mathrm{p}<0.001$; Figure $2 \mathrm{C}$ ), while sperm motility grade $\mathrm{D}$ was significantly higher ( $p<0.001$; Figure 2D) for the group of RT carriers in comparison to the control subjects. No significant differences in progressive sperm motility and immotile sperm were observed between the RCT carriers and control group.

\section{Discussion}

We present a study of the semen of a large group of balanced somatic translocation carriers and men 
Table 1. Patients characteristics, semen and sperm parameters of balanced translocation carriers and control subjects with normal karyotype

\begin{tabular}{|c|c|c|c|c|}
\hline & $\begin{array}{l}\text { Normal karyotype } \\
\text { (control group; } \\
\text { n = 57) }\end{array}$ & $\begin{array}{c}\text { Reciprocal } \\
\text { translocations } \\
(\mathrm{RCT} ; \mathbf{n}=49) \\
\end{array}$ & $\begin{array}{c}\text { Robertsonian } \\
\text { translocations } \\
(\mathrm{RT} ; \mathbf{n}=\mathbf{3 5})\end{array}$ & $\mathbf{p}^{*}$ \\
\hline Age (years) & $36.37 \pm 4.16$ & $34.65 \pm 4.72$ & $35.03 \pm 4.49$ & 0.059 \\
\hline Sexual abstinence (day) & $5.29 \pm 2.82$ & $4.63 \pm 4.74$ & $4.52 \pm 1.39$ & 0.717 \\
\hline Seminal volume $[\mathrm{mL}]$ & $3.75 \pm 1.77$ & $3.38 \pm 1.80$ & $3.89 \pm 2.09$ & 0.368 \\
\hline Sperm concentration [million/mL] & $49.87 \pm 44.58^{b}$ & $35.23 \pm 35.51^{\mathrm{b}}$ & $15.22 \pm 25.83^{\mathrm{a}}$ & $<0.001$ \\
\hline $\begin{array}{l}\text { Normozoospermia }(\mathrm{n}=78) \\
(>15 \mathrm{million} / \mathrm{mL})\end{array}$ & $71.9 \%(41)$ & $57.1 \%(28)$ & $25.7 \%(9)$ & \\
\hline $\begin{array}{l}\text { Abnormal sperm concentration }(\mathrm{n}=63) \\
(<15 \mathrm{million} / \mathrm{mL})\end{array}$ & $28.1 \%(16)$ & $42.9 \%(21)$ & $74.3 \%(26)$ & \\
\hline Azoospermia $(n=4)$ & $0 \%(0)$ & $6.1 \%(3)$ & $2.9 \%(1)$ & 0.001 \\
\hline Cryptozoospermia $(\mathrm{n}=6)$ & $1.8 \%(1)$ & $4.1 \%(2)$ & $8.6 \%(3)$ & \\
\hline Severe oligozoospermia $(<5$ million $/ \mathrm{mL})(\mathrm{n}=24)$ & $7.0 \%(4)$ & $18.4 \%(9)$ & $31.4 \%(11)$ & \\
\hline Oligozoospermia $(5-15$ million/mL) $(\mathrm{n}=29)$ & $19.3 \%(11)$ & $14.3 \%(7)$ & $31.4 \%(11)$ & \\
\hline \multicolumn{5}{|l|}{ Sperm motility (\%) } \\
\hline$A+B$ & $39.77 \pm 19.40^{\mathrm{b}}$ & $32.47 \pm 21.03^{\mathrm{ab}}$ & $21.53 \pm 18.37^{\mathrm{a}}$ & $<0.001$ \\
\hline $\mathrm{C}$ & $12.88 \pm 8.60$ & $12.33 \pm 9.07$ & $12.67 \pm 10.37$ & 0.912 \\
\hline $\mathrm{D}$ & $47.34 \pm 21.76^{\mathrm{a}}$ & $55.23 \pm 25.25^{\mathrm{ab}}$ & $66.23 \pm 20.28^{\mathrm{b}}$ & 0.002 \\
\hline Sperm vitality: live (\%) & $77.47 \pm 13.03^{b}$ & $76.17 \pm 15.84^{\mathrm{b}}$ & $62.17 \pm 18.78^{\mathrm{a}}$ & $<0.001$ \\
\hline Round cell [million/mL] & $3.42 \pm 5.71$ & $1.89 \pm 1.20$ & $1.98 \pm 1.44$ & 0.555 \\
\hline DNA fragmentation index (\%) & $13.86 \pm 5.49$ & $12.47 \pm 6.34$ & $15.78 \pm 7.06$ & 0.194 \\
\hline
\end{tabular}

Data are mean $\pm \mathrm{SD}$ or prevalence, $\mathrm{n}$ is given in parentheses. ${ }^{*}$ Comparison between groups was performed by Kruskal-Wallis test for continuous data and by the $\chi^{2}$ test for categorical data. Different letters $\left({ }^{(a, b}\right)$ indicate statistically significant differences (performed by post-hoc Dunn's Bonferroni test) within the RCT, RT and control groups. DNA fragmentation was determined by the TUNEL technique as described in Material and methods

with normal karyotype to establish sperm parameters. We compared retrospectively results obtained from analysis of the semen samples which were not frozen before tests and were categorized in a homogeneous groups of the RCT and RT translocation carriers. The use of semen samples from donors with normal karyotype who were partners of the female with established etiology of infertility, provided us with a reliable control group.

Probability of detecting a chromosomal rearrangements in a patient's karyotype is negatively correlated to the sperm concentration [14-17]. Indeed, in this study, we observed lowered sperm concentration in the RT carrier group, but not in the RCT group in comparison to the control subjects. Significantly lower concentrations of sperm in semen of RT translocation carriers in comparison to ejaculated samples of fertile donors were also observed by other authors [18]. The lower number of sperm in semen of RT carriers can be the result of spermatogenesis impairment caused by the process of segregation and improper gene ex- pression during gamete formation [19, 20]. Activation of the process of apoptosis which allowed, at least partially, for exclusion of cells with chromosomal rearrangements [21] during spermiogenesis [18], can also explain this phenomenon.

In our study a number of RT $(25.7 \%)$ and RCT $(57.1 \%)$ carriers had sperm parameters within normal ranges. However, those parameters are lower than the rates mentioned in studies by Vozdova et al. $(30.8 \%$ of RT and 59.5\% of RCT translocation carriers) [22] and the data presented by other authors [19]. This suggests that autosomal rearrangement of the karyotype does not always lead to severe oligozoospermia or azoospermia as do sex chromosome aberrations [5].

Chandley et al. [23] showed a reduced proportion of spermatids and spermatozoa on testicular biopsy in subfertile balanced translocations carriers. Balanced, structural chromosome aberrations seldom result in azoospermia [20, 24]. The most often reported phenotypes for balanced translocation carriers usually vary from severe oligozoospermia to normozoospermia [2, 

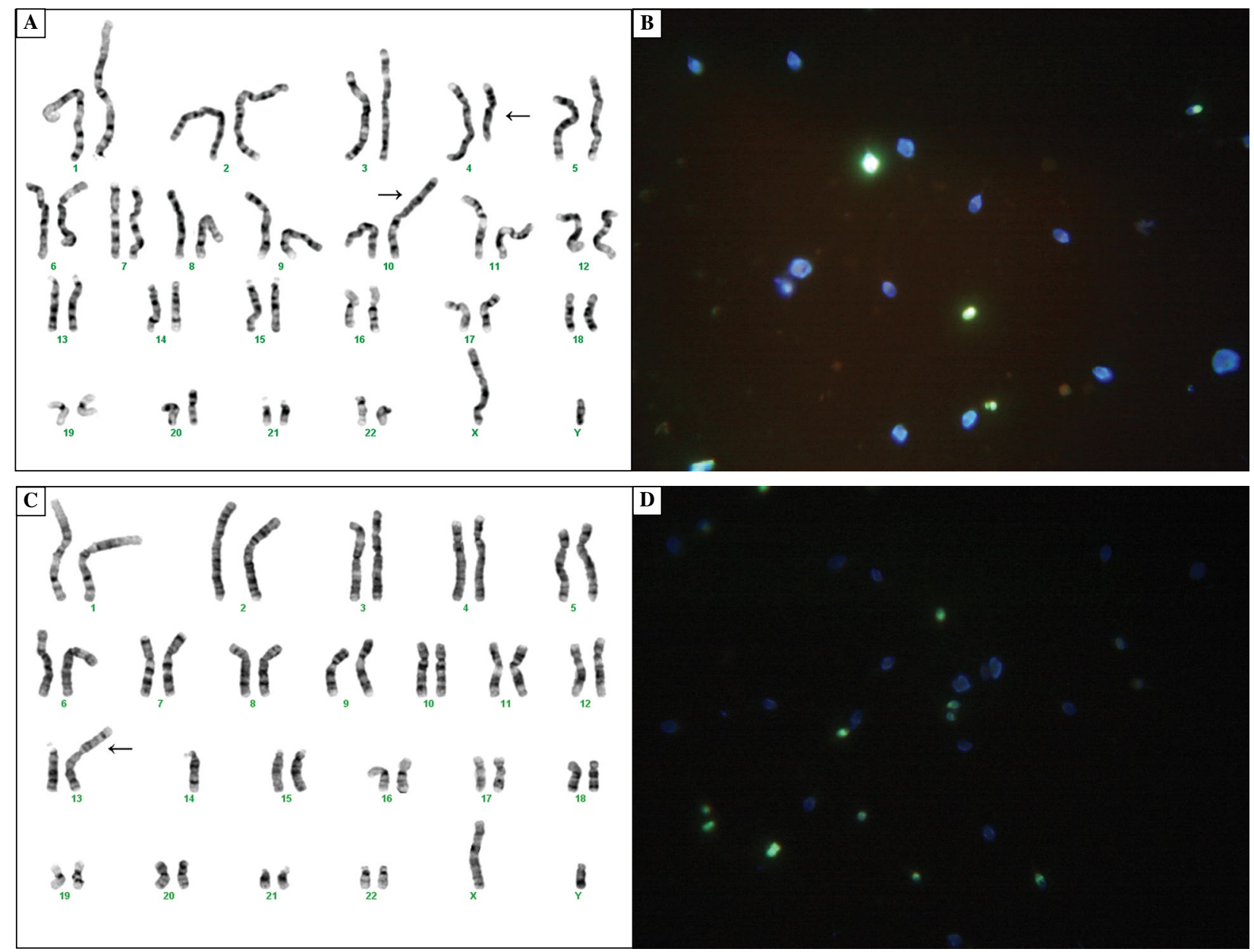

Figure 1. Exemplary lymphocytic karyogram of the patients carrying RCT (46,XY,t(4;10)(q21;p11.2) (A), and RT $(45, X Y, \operatorname{der}(13 ; 14)(q 10 ; q 10))(\mathbf{C})$ translocations. TUNEL analysis of spermatozoa of the same patients carrying RCT (B) and RT (D) translocations. Magnification: karyogram $-\times 1000$; TUNEL analysis $-\times 630$

$20,25]$. Nonetheless, in our study we found statistically significant difference between sperm concentration and occurrence of chromosomal rearrangements. This parameter in RT translocation carriers was lower than in the other groups. At the same time, most aberrations are found in azoospermic and severely oligozoospermic men [9] what is partially in agreement with the results of our study. However, other authors did observe such high correlation in subgroups of infertile men $[26,27]$.

In our study, we showed decreased progressive sperm motility and increased sperm immotility in RT carriers. It was suggested that unbalanced chromosomal status has no impact on the motility of the sperm [28]. Other authors presented results showing no differences in terms of this sperm parameter between men with normal and abnormal karyotypes [29, 30]. On the other hand, comparisons of RT carriers and control donors revealed decreased sperm motility in the group of patients carrying RT $[18,19,22]$ for those with normal [19] and abnormal semen parameters [22]. Additionally, more recent data show only $4 \%$ progressive motility in $45, \mathrm{XY}, \mathrm{t}(14 ; 14)(\mathrm{q} 10 ; \mathrm{q} 10)$ patients [31] and $8-17 \%$ progressive motility in $45, \mathrm{XY}, \mathrm{t}(14 ; 22)$ patients [32]. Fadlalla et al. [24] presented decreased sperm motility in $45, \mathrm{XY}, \operatorname{der}(13 ; 14)$ (q11;p11) donors, while Morel et al. [33] showed semen parameters of three $45, \mathrm{XY}$, der(13;14)(q10;q10) patients whose sperm motility were significantly reduced. In our studies 24 out of 35 RT carriers were 45,XY, der(13;14)(q10;q10). However, we did not find difference between $45, \mathrm{XY}$, der(13;14) versus others RT carriers (data not shown). Moreover, the influence of freezing on diminished sperm motility [34] can be excluded, as we retrospectively considered only the results of analyses carried out on a fresh semen samples.

Results of our investigation showed that in all of studied group sperm vitality was not lower than $58 \%$, what is the WHO standard and what is in agreement with the data presented by others [32, 35]. However, 


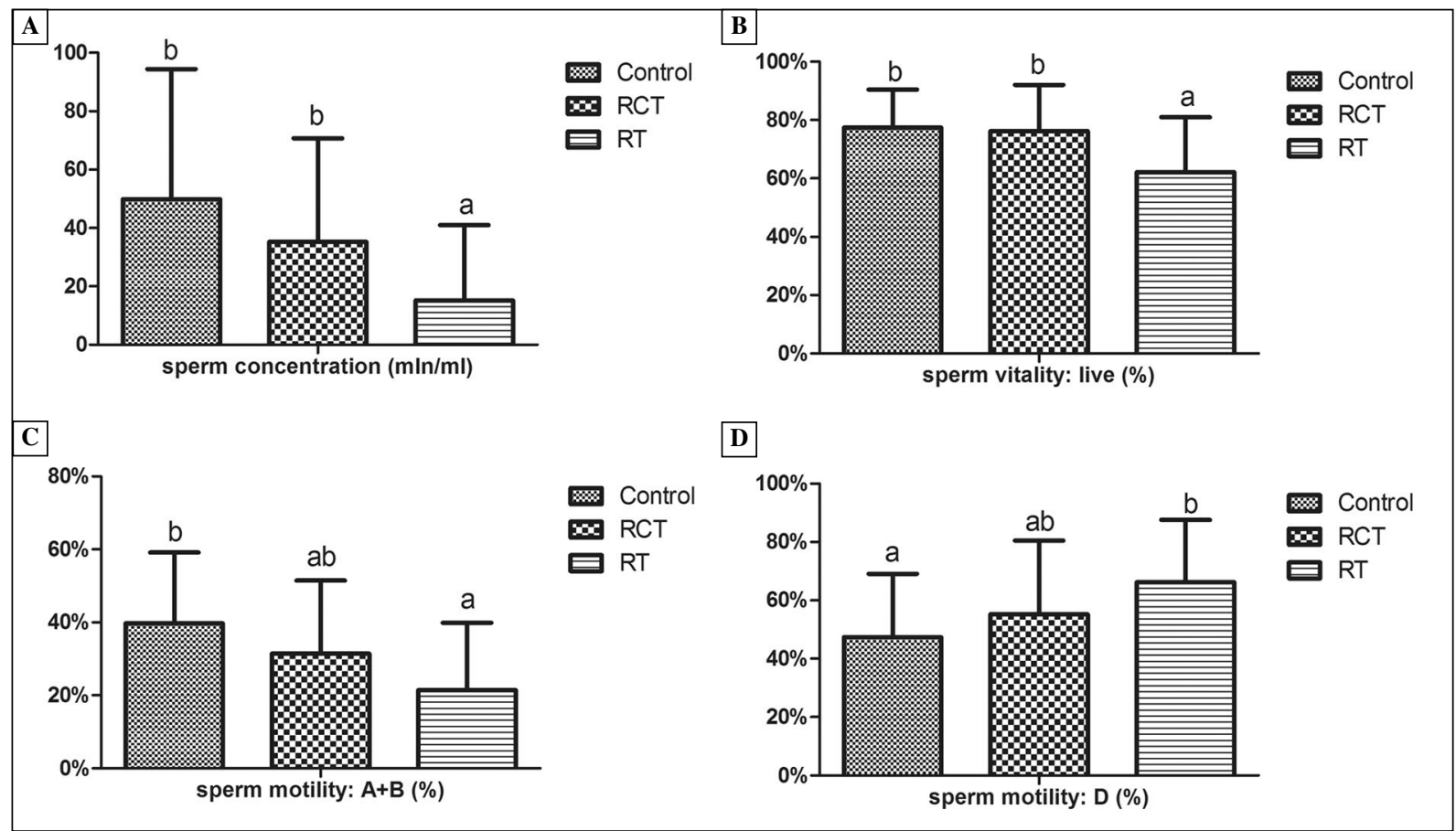

Figure 2. Characteristics of sperm of control, RCT and RT carriers. Concentration (A), vitality (B), progressive motility grade A + B (C), motility grade D (D). Different letters indicate statistically significant differences (performed by post-hoc Dunn's Bonferroni test) within the RCT, RT and control groups

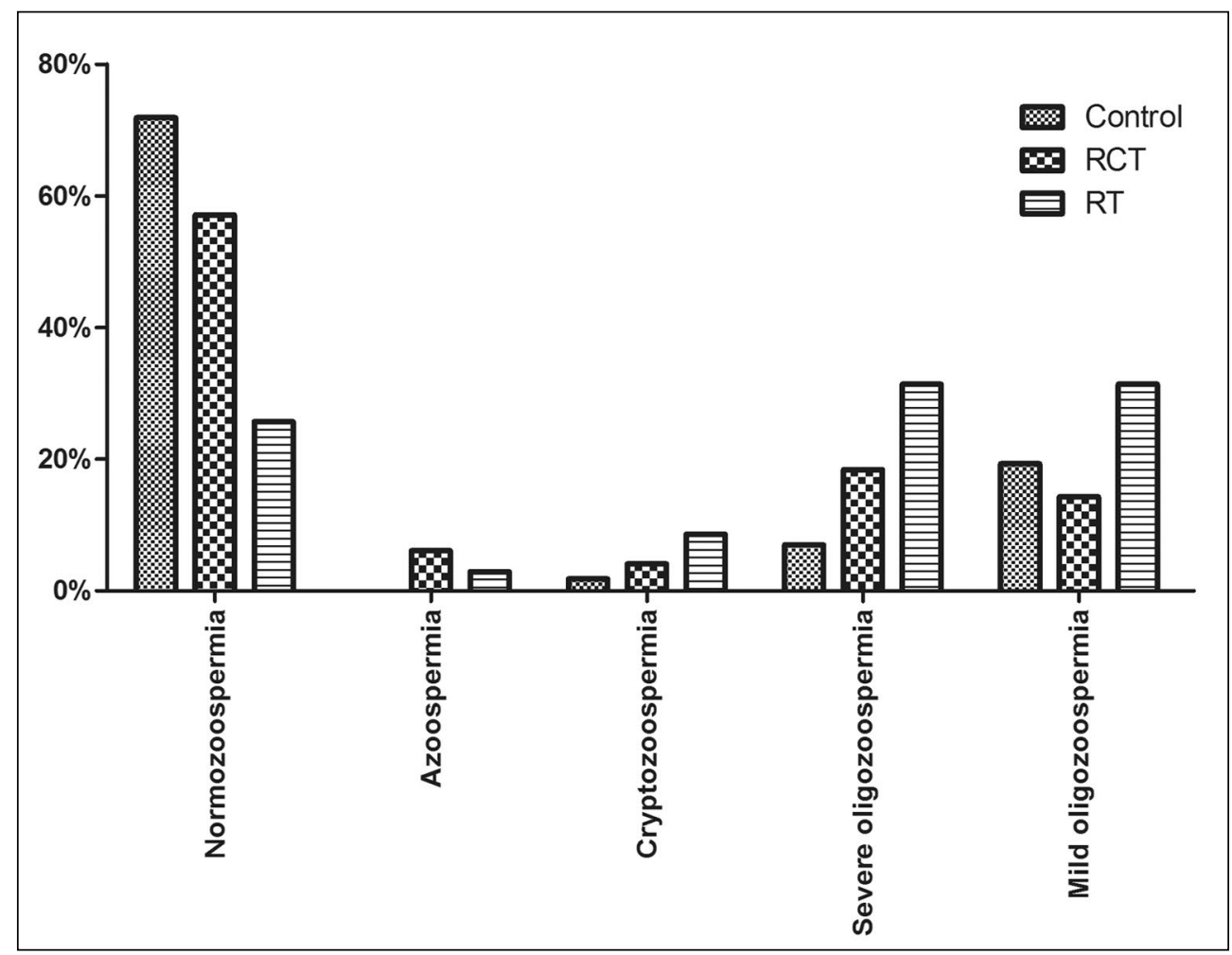

Figure 3. Relative frequency of distribution of occurrences of normal/abnormal sperm concentration in control, RCT and RT groups. Values are expressed as \% of study groups 
our study additionally presented that in RT carriers these values were reduced in comparison to both control and RCT group. As far as we know until now, there has not been any publication presenting such data.

Sperm DNA fragmentation designated as DNA fragmentation index reflects apoptotic process which takes place in a cell. The DNA damage rate, which may be affected by age [36] is significantly higher in the ejaculated sperm of translocation carriers than in control subjects $[18,37,38]$. Moreover, DNA fragmentation index is higher in spermatozoa of RT carriers with chromosomally unbalanced content in comparison to those who have normal or balanced ejaculated spermatozoa [21]. Thus, apoptosis seems to be quite common for spermatozoa of translocation carriers as it can function as a mechanism of selection of abnormal spermatozoa. In male germ cells, meiotic spindle check-point mechanism, as a guard of proper meiosis, is present. If aberrant meiosis occurs, activation of apoptosis takes place. Moreover, oxidative stress can also evoke activation of this process. Taking all of the above mentioned aspects into the consideration, the results of our study at this point are inconsistent with the existing publications. We showed only slightly higher DNA fragmentation index in RT and slightly lower DNA fragmentation index in RCT patients, but we did not observed any statistical significance. We assumed that the reason is the fact that in most studies [18, 38-40] control population consisted of donors whose sperm parameters meet WHO criteria. In our study, we included donors whose semen not only met the WHO criteria but also had a population distribution similar to that of male factor, what probably had influenced our results. The presented results clearly point out that the semen of RT carriers contained fewer spermatozoa, had impaired motility and lowered vitality. The risks of passing RT chromosomal rearrangements to the next generation put emphasis on the importance of careful evaluation of semen. Carriers of mainly RT but also RCT have an increased risk of not only poorer semen quality but also infertility, repeated miscarriages and conception of offspring with unbalanced karyotypes. Therefore thorough semen analysis combined with karyotyping should be considered as part of genetic counseling. Such genetic counseling should be an important part of the infertility treatment process that would lead to improvement of outcomes for patients who are translocation carriers.

\section{References}

1. Ferlin A, Raicu F, Gatta V, Zuccarello D, Palka G, Foresta C. Male infertility: role of genetic background. Reprod Biomed Online. 2007;14:734-745. PMID: 17579990.
2. O'Flynn O'Brien KL, Varghese AC, Agarwal A. The genetic causes of male factor infertility: a review. Fertil Steril. 2010;93:1-12. doi: 10.1016/j.fertnstert.2009.10.045.

3. Poongothai J, Gopenath TS, Manonayaki S. Genetics of human male infertility. Singapore Med J. 2009;50:336-347. PMID: 19421675.

4. Piomboni P, Stendardi A, Gambera L. Chromosomal aberrations and aneuploidies of spermatozoa. Adv Exp Med Biol. 2014;791:27-52. doi: 10.1007/978-1-4614-7783-9 3.

5. Martin RH. Cytogenetic determinants of male fertility. Hum Reprod Updat. 2008;14:379-390. doi: 10.1093/humupd/ dmn017.

6. Harton GL, Tempest HG. Chromosomal disorders and male infertility. Asian J Androl. 2012;14:32-39. doi: 10.1038/ aja.2011.66.

7. Ceylan GG, Ceylan C, Elyas H. Genetic anomalies in patients with severe oligozoospermia and azoospermia in eastern Turkey: a prospective study. Genet Mol Res. 2009;8:915-922. doi: 10.4238/vol8-3gmr616.

8. Balkan M, Tekes S, Gedik A. Cytogenetic and Y chromosome microdeletion screening studies in infertile males with oligozoospermia and azoospermia in southeast Turkey.J Assist Reprod Gen. 2008;25:559-565. doi: 10.1007/s10815-008-9272-8.

9. Dul EC, van Ravenswaaij-Arts CMA, Groen H, van Echten-Arends J, Land JA. Who should be screened for chromosomal abnormalities before ICSI treatment? Hum Reprod. 2010;25:2673-2677. doi: 10.1093/humrep/deq258.

10. Shamsi MB, Kumar R, Malhotra N et al. Chromosomal aberrations, Yq microdeletion, and sperm DNA fragmentation in infertile men opting for assisted reproduction. Mol Reprod Dev. 2012;79:637-650. doi: 10.1002/mrd.22072.

11. Celep F, Karagüzel A, Ozeren M, Bozkaya H. The frequency of chromosomal abnormalities in patients with reproductive failure. Eur J Obstet Gynecol Reprod Biol. 2006;127:106-109. doi: 10.1016/j.ejogrb.2005.12.019.

12. World Health Organization. WHO Laboratory Manual for the Examination of Human Semen and Sperm Cervical Mucu Interaction. $5^{\text {th }}$ ed. Cambridge University Press; 2010.

13. Bangs CD, Donlon TA. Metaphase chromosome preparation from cultured peripheral blood cells. Curr Protoc Hum Genet. 2005; Chapter 4:Unit 4.1. doi: 10.1002/0471142905.hg0401s45.

14. Clementini E. Prevalence of chromosomal abnormalities in 2078 infertile couples referred for assisted reproductive techniques. Hum Reprod. 2004;20:437-442. doi: 10.1093/ humrep/deh626.

15. Koşar PA, Ozçelik N, Koşar A. Cytogenetic abnormalities detected in patients with non-obstructive azoospermia and severe oligozoospermia.J Assist Reprod Genet. 2010;27:17-21. doi: 10.1007/s10815-009-9366-y.

16. Abramsson L, Beckman G, Duchek M, Nordenson I. Chromosomal aberrations and male infertility.J Urol. 1982;128:52-53. PMID: 7109070.

17. Chandley AC. The chromosomal basis of human infertility. Br Med Bull. 1979;35:181-186. PMID: 387165.

18. Brugnon F, Janny L, Communal Y et al. Apoptosis and meiotic segregation in ejaculated sperm from Robertsonian translocation carrier patients. Hum Reprod. 2010;25:1631-1642. doi: 10.1093/humrep/deq113.

19. Ferfouri F, Selva J, Boitrelle F et al. The chromosomal risk in sperm from heterozygous Robertsonian translocation carriers is related to the sperm count and the translocation type. Fertil Steril. 2011;96:1337-1343. doi: 10.1016/j.fertnstert.2011.09.008.

20. Dong Y, Du R-C, Jiang Y-T, Wu J, Li L-L, Liu R-Z. Impact of chromosomal translocations on male infertility, semen 
quality, testicular volume and reproductive hormone levels. J Int Med Res. 2012;40:2274-2283. PMID: 23321184.

21. Perrin A, Nguyen MH, Bujan Let al. DNA fragmentation is higher in spermatozoa with chromosomally unbalanced content in men with a structural chromosomal rearrangement. Andrology. 2013;1:632-638. doi: 10.1111/j.2047-2927.2013.00100.x.

22. Vozdova M, Oracova E, Kasikova $\mathrm{K}$ et al. Balanced chromosomal translocations in men: relationships among semen parameters, chromatin integrity, sperm meiotic segregation and aneuploidy. J Assist Reprod Genet. 2013;30:391-405. doi: 10.1007/s10815-012-9921-9.

23. Chandley AC, Maclean N, Edmond P, Fletcher J, Watson GS. Cytogenetics and infertility in man. II. Testicular histology and meiosis. Ann Hum Genet. 1976;40:165-176. PMID: 1015811.

24. Elfateh F, Wang R, Zhang Z, Jiang Y, Chen S, Liu R. Influence of genetic abnormalities on semen quality and male fertility: A four-year prospective study. Iran J Reprod Med. 2014;12:95-102. PMID: 24799866.

25. Jungwirth A, Giwercman A, Tournaye H et al. European Association of Urology guidelines on Male Infertility: the 2012 update. Eur Urol. 2012;62:324-332. doi: 10.1016/j. eururo.2012.04.048

26. Dul EC, Groen H, van Ravenswaaij-Arts CMA, Dijkhuizen T, van Echten-Arends J, Land JA. The prevalence of chromosomal abnormalities in subgroups of infertile men. Hum Reprod. 2012;27:36-43. doi: 10.1093/humrep/der374.

27. Riccaboni A, Lalatta F, Caliari I, Bonetti S, Somigliana E, Ragni G. Genetic screening in 2,710 infertile candidate couples for assisted reproductive techniques: results of application of Italian guidelines for the appropriate use of genetic tests. Fertil Steril. 2008;89:800-808. doi: 10.1016/j. fertnstert.2007.04.032.

28. Vozdova M, Kasikova K, Oracova E et al. The effect of the swim-up and hyaluronan-binding methods on the frequency of abnormal spermatozoa detected by FISH and SCSA in carriers of balanced chromosomal translocations. Hum Reprod. 2012;27:930-937. doi: 10.1093/humrep/der445.

29. Yoshida A, Miura K, Shirai M. Cytogenetic survey of 1,007 infertile males. Urol Int. 1997;58:166-176. PMID: 9188138.

30. Gekas J, Thepot F, Turleau C et al. Chromosomal factors of infertility in candidate couples for ICSI: an equal risk of constitutional aberrations in women and men. Hum Reprod. 2001;16:82-90. PMID: 11139542.
31. Cinar C, Beyazyurek C, Ekmekci CG, Aslan C, Kahraman $\mathrm{S}$. Sperm fluorescence in situ hybridization analysis reveals normal sperm cells for 14;14 homologous male Robertsonian translocation carrier. Fertil Steril. 2011;95:289.e5-9. doi: 10.1016/j.fertnstert.2010.05.033.

32. Baccetti B, Capitani S, Collodel G, Estenoz M, Gambera L, Piomboni P. Infertile spermatozoa in a human carrier of robertsonian translocation 14;22. Fertil Steril. 2002;78:1127-1130. PMID: 12414006.

33. Morel F, Roux C, Bresson JL. FISH analysis of the chromosomal status of spermatozoa from three men with 45,XY,der(13;14)(q10;q10) karyotype. Mol Hum Reprod. 2001;7:483-488. PMID: 11331672.

34. Punyatanasakchai $P$, Sophonsritsuk A, Weerakiet S, Wansumrit S, Chompurat D. Comparison of cryopreserved human sperm in vapor and liquid phases of liquid nitrogen: effect on motility parameters, morphology, and sperm function. Fertil Steril. 2008;90:1978-1982. doi: 10.1016/j. fertnstert.2007.09.066.

35. Baccetti B. Ultrastructural studies of spermatozoa from infertile males with Robertsonian translocations and 18, X, Y aneuploidies. Hum Reprod. 2005;20:2295-2300. doi: 10.1093/ humrep/dei050.

36. Bojar I, Witczak M, Wdowiak A. Biological and environmental conditionings for a sperm DNA fragmentation. Ann Agric Environ Med. 2013;20:865-868. PMID: 24364470.

37. Perrin A, Basinko A, Douet-Guilbert N et al. Aneuploidy and DNA fragmentation in sperm of carriers of a constitutional chromosomal abnormality. Cytogenet Genome Res. 2011;133:100-106. doi: 10.1159/000323980.

38. Perrin A, Caer E, Oliver-Bonet $\mathrm{M}$ et al. DNA fragmentation and meiotic segregation in sperm of carriers of a chromosomal structural abnormality. Fertil Steril. 2009;92:583-589. doi: 10.1016/j.fertnstert.2008.06.052.

39. Brugnon F, Van Assche E, Verheyen G et al. Study of two markers of apoptosis and meiotic segregation in ejaculated sperm of chromosomal translocation carrier patients. Hum Reprod. 2006;21:685-693. doi: 10.1093/humrep/dei401.

40. Olszewska M, Fraczek M, Huleyuk N et al. Chromatin structure analysis of spermatozoa from reciprocal chromosome translocation (RCT) carriers with known meiotic segregation patterns. Reprod Biol. 2013;13:209-220. doi: 10.1016/j. repbio.2013.06.002. 